Preprint version of an article published in: International Journal of Computer Integrated Manufacturing (IJCIM), Volume 17, Issue 3, April 2004, pages 254-264, DOI:10.1080/09511920310001607069, (c) Taylor \& Francis

\title{
Building an E-Marketplace on a Peer-to-Peer Infrastructure
}

\author{
Diogo R. Ferreira ${ }^{1}$, J. J. Pinto Ferreira ${ }^{2}$ \\ ${ }^{1}$ INESC Porto, Campus FEUP, Rua Dr. Roberto Frias, $n^{\circ}$ 378, 4200-465 Porto, Portugal \\ ${ }^{2}$ Faculty of Engineering U. P., Rua Dr. Roberto Frias s/n, 4200-465 Porto, Portugal \\ \{drf@inescporto.pt,jjpf@fe.up.pt\}
}

\begin{abstract}
Existing e-marketplaces, built on traditional client-server architectures, severely restrict the scope and dynamics of Business-to-Business (B2B) interactions. Peer-to-peer (P2P) architectures will provide far more decentralised infrastructures, while allowing a much wider range of business patterns to take place. On one hand, the interaction over a P2P network resembles the way real-world enterprises perform business with each other. On the other hand, a small set of simple services is enough to support complex business processes over a P2P infrastructure. Incidentally, most of the required technology is readily available, though it may be necessary to bring in an appropriate integration of different concepts. The paper discusses the implementation of essential services for P2P e-marketplaces, based on one of the leading P2P platforms, and illustrates its benefits by applying the P2P approach to a vendor of industrial equipment.
\end{abstract}

\section{Introduction}

The rise of global and ubiquitous infrastructures, namely the Internet and the World Wide Web, has brought speed and connectivity to the whole spectrum of Business-toBusiness (B2B) exchanges, from simple document delivery to complete and automated trading procedures. E-business possibilities have led to new B2B models, particularly the emarketplace.

Like other business models, the e-marketplace is hardly mentioned today, though once it was thought of as the next big thing. Even if many e-marketplaces have disappeared, most buyers and suppliers would still be eager to identify and exploit new business opportunities. However, the traditional concept of e-marketplace supports a single business model, which is ineffective in dealing with all but the simplest kinds of exchanges. As a result, the traditional concept of an e-marketplace as a broker mediating between buyer and supplier is not suitable for every kind of product transactions, as it was initially expected.

This paper explains why the traditional e-marketplace is not entirely suitable for B2B exchanges and proposes a peer-to-peer (P2P) infrastructure in order to overcome these pitfalls. Currently available P2P platforms still lack essential services before they can be applied in a business environment. These services must support the full B2B trading life-cycle from partner selection to contract fulfilment. This paper reports the implementation of the services that support the B2B trading life-cycle, using one of the leading $\mathrm{P} 2 \mathrm{P}$ platforms, and the use of these services in an industrial equipment e-marketplace.

\section{The traditional e-marketplace}

The traditional e-marketplace is a Web portal where buyers and suppliers come together to explore new business opportunities. There are three main types of e-marketplace (Norris 2001): the seller-driven market is an e-marketplace promoted by a consortium of 
suppliers who place offers within the same industry or service sector, the buyer-driven market is maintained by a group of buyers who aggregate purchase needs so as to achieve advantageous conditions when buying from suppliers, and the open market is an emarketplace owned by an independent third-party.

The goal of the e-marketplace is to attract the biggest possible number of buyers and suppliers, which will become members of that e-marketplace. Buyers bring purchase needs while suppliers bring selling offers, as suggested in figure 1 . The e-marketplace will then match purchase needs against selling offers, encouraging its members to undertake new trading exchanges. Typically, a prospective supplier publishes offers while a prospective buyer searches for products or services by looking up offers on the marketplace database. If an interesting offer is found, the entity running the e-marketplace will work as a broker, mediating the contact between buyer and supplier. Buyer and supplier will typically interact solely through the e-marketplace, and never directly. The broker will usually charge a monthly fee or a percentage of the transaction value.

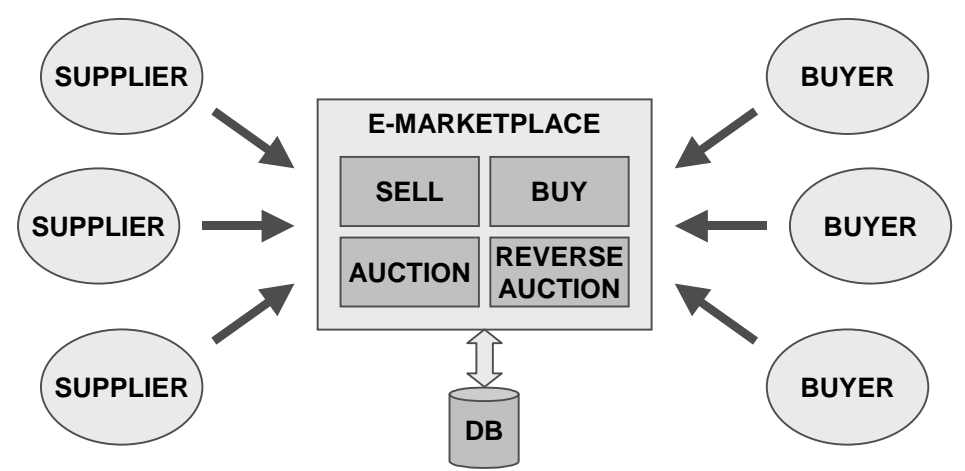

Figure 1. Architecture of the traditional e-marketplace

Every e-marketplace thus comprises two main areas, as shown in figure 1, commonly referred to as the supplier side and the buyer side. The supplier side is the entry point for suppliers, where they can advertise their selling offers. In the buyer side, buyers advertise purchase needs. Besides matchmaking, the e-marketplace usually provides other services such as auction (where a supplier sells to the buyer who submits the highest bid for a given selling offer), and reverse auction (where a buyer buys from the supplier who submits the lowest bid for a given purchase need). The e-marketplace is the central hub providing all these services.

It can therefore be argued that the traditional e-marketplace exhibits the characteristics shown in table 1. 


\begin{tabular}{|c|c|}
\hline Centralised repository & $\begin{array}{l}\text { The traditional e-marketplace is a centralised information repository where } \\
\text { buyers and suppliers can publish and retrieve information of their interest. }\end{array}$ \\
\hline Opaque interfacing & $\begin{array}{l}\text { Although buyers and suppliers can browse selling offers or purchase needs } \\
\text { and can initiate and perform trading exchanges, the e-marketplace will not } \\
\text { reveal the trading partners' identity in order to ensure its intermediary role. }\end{array}$ \\
\hline Static information & $\begin{array}{l}\text { Because a buyer retrieves a selling offer that a supplier has published in the } \\
\text { e-marketplace database, there is no way for a supplier to immediately notify } \\
\text { all potential buyers of dynamic conditions such as a sales campaign or a } \\
\text { temporary shortage of product items. }\end{array}$ \\
\hline Bottlenecked structure & $\begin{array}{l}\text { All information submissions, retrievals, and trading exchanges must go } \\
\text { through the same e-marketplace hub. If the e-marketplace malfunctions or } \\
\text { disappears, all of its information, transactions and opportunities are at risk. }\end{array}$ \\
\hline Loosely connected & $\begin{array}{l}\text { The e-marketplace enables buyers and suppliers to perform trading } \\
\text { exchanges, but does not allow these members to group together or build } \\
\text { virtual communities. }\end{array}$ \\
\hline Fixed exchanges & $\begin{array}{l}\text { There are predefined data formats and rules for performing exchanges, and } \\
\text { the e-marketplace does not tolerate deviations from or augmentations to } \\
\text { these exchange rules. }\end{array}$ \\
\hline Price-based decisions & $\begin{array}{l}\text { When looking for a supplier to fulfil a particular purchase need, the buyer is } \\
\text { presented with little more than the price according to each possible supplier. } \\
\text { Choosing the supplier with the lowest unit cost may not yield the best result. }\end{array}$ \\
\hline Non-iterated agreements & $\begin{array}{l}\text { Because exchanges are opaque, fixed and price-based, there is no support } \\
\text { for agreements attained by iterated phases of negotiation with proposals and } \\
\text { counter-proposals. }\end{array}$ \\
\hline Short-term partnerships & $\begin{array}{l}\text { After the exchange is complete, the buyer will have no further information } \\
\text { on the supplier other than how well the supplier has performed, and } \\
\text { absolutely no information about how other suppliers in the e-marketplace } \\
\text { are performing. }\end{array}$ \\
\hline Service charges & $\begin{array}{l}\text { The e-marketplace lives on the premise of bringing together buyers and } \\
\text { suppliers so as to increase the volume of exchanges, and to provide its } \\
\text { members with an increasing number of business opportunities. However, } \\
\text { service fees may be dissuading for prospective members. }\end{array}$ \\
\hline
\end{tabular}

Table 1. Characteristics of traditional e-marketplaces

The traditional e-marketplace displays some advantages. For example, the presence of the e-marketplace as an intermediary ensures that the supplier will be paid for the delivered goods, even if the supplier does not know if the buyer is trustworthy or not. In this case, the emarketplace guarantees the reliability of transactions: members trust the e-marketplace without having necessarily to trust other members. This concept may be somewhat counterintuitive from a business perspective, but is difficult to circumvent in any kind of emarketplace. It may be regarded as deductive trust, as discussed later in section 3.5.

The characteristics of the traditional e-marketplace point to a number of drawbacks that can ultimately explain why this business model has proved to be ineffective. But then, given that the traditional e-marketplace displays these serious drawbacks, the question is whether there is another kind of e-marketplace that overcomes some of these pitfalls. If so, which are the services this new kind of e-marketplace should provide? And how can these services be implemented? In essence, how to build a new kind of e-marketplace that better supports the way companies perform business with each other?

\section{The P2P e-marketplace}

The services provided by an e-marketplace may be approached from a peer-to-peer (P2P) perspective, bringing numerous advantages. On a P2P e-marketplace, each peer node represents a buyer or a supplier, and each peer is connected to every other peer, either directly 
or through other peer nodes, as shown in figure 2. Peers may search, connect, and exchange data with other peers. In a P2P network, suppliers may publish or advertise information about their offers. These suppliers rely on the P2P infrastructure to convey that information to other peers. Buyers, on the other hand, rely on P2P search capabilities to locate and retrieve offers, and on the network infrastructure itself to connect and interact with suppliers.

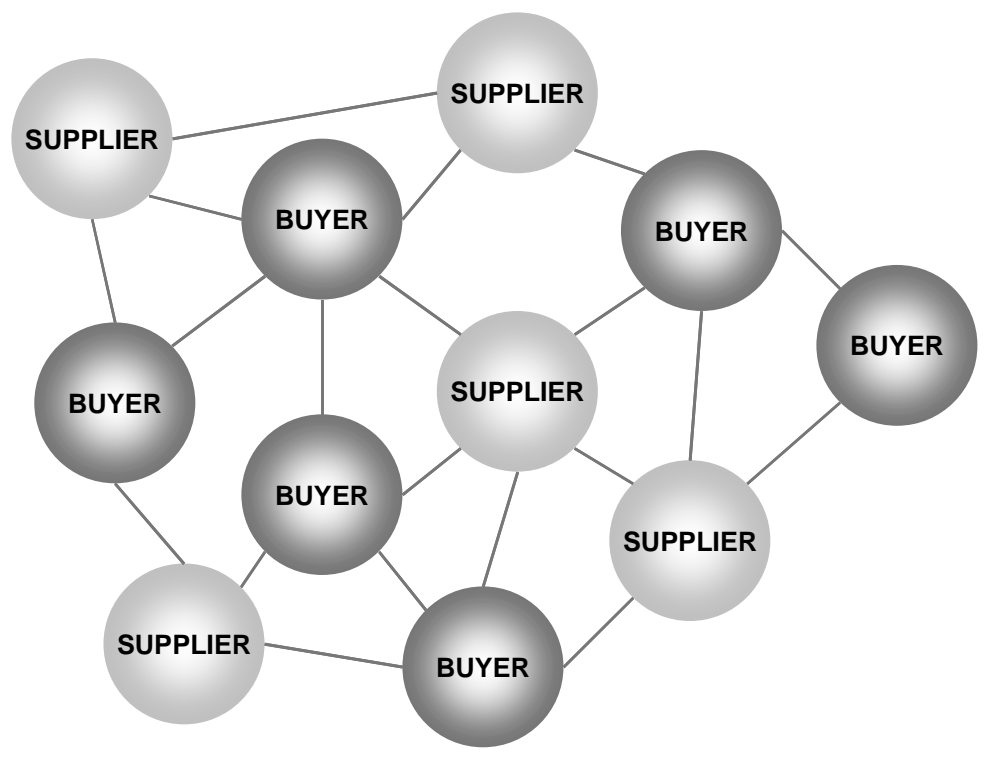

Figure 2. The P2P e-marketplace

When compared to the traditional e-marketplace, the P2P e-marketplace overcomes the previous disadvantages:

- It is not a centralised repository but a completely distributed one;

- Peers are able to interact directly and perform one-to-one exchanges;

- Up-to-date, dynamic information can be provided by one peer to another;

- Transactions are distributed across the network and the addition or removal of one node does not put the e-marketplace at risk; instead, it shapes the e-marketplace;

- Peers can attach to each other and develop virtual communities;

- The exchange rules can be set by each pair of peers performing an exchange;

- Peers can exchange virtually any information in order to make decisions;

- Peers are able to settle agreements by iterated phases of negotiation;

- Each peer is able to collect more information about other peers and about the e-marketplace in general, so as to develop the most advantageous, possibly long-term relationships; and

- It is possible to set up a P2P e-marketplace without service charges, where everyone benefits from being able to connect to everyone else.

Up to now, interaction over P2P networks has been usually confined to two phases, as depicted in figure 3(a) and figure 3(b): The search-and-find phase when one peer contacts several others in order to locate desired information; and the connect-and-retrieve phase when the same peer obtains the intended data from another peer. These two basic P2P networking mechanisms - one-to-many (or multicast) search and one-to-one (or unicast) transfer - open a wide range of possibilities for more complex business patterns, limited only by the breadth of functionality that peers are able to implement. 


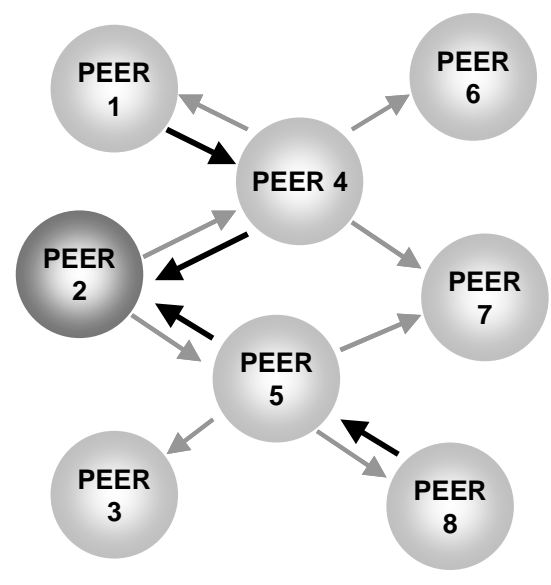

(a)

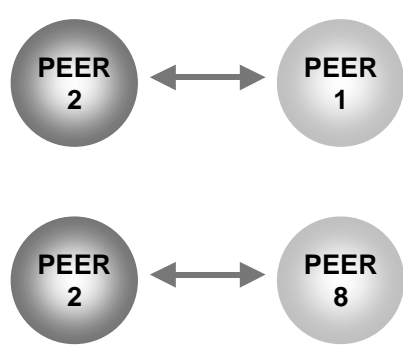

(b)

Figure 3. Multicast search (a) and unicast retrieval (b) over a P2P network

For the purpose of P2P e-marketplaces, however, these two mechanisms are still limited, because peers must be able to advertise selling offers and purchase needs, to conduct searches for those opportunities, and to interact with other peers, for example while negotiating trading conditions or when performing the agreed exchanges. The solution for such an interoperable and extensible networking platform lies in adopting a layered approach: the P2P e-marketplace is required to implement a set of essential services that will allow other, more complex services to be built on top of those services. But what should these essential services be and how should they be implemented? To answer these questions, one must delve into the context and requirements of business-to-business trading.

\section{The B2B trading life-cycle}

Business-to-business transactions can be regarded as being comprised of five main phases, i.e., the search phase, the selection phase, the contracting phase, the fulfilment phase, and the evaluation phase. This life-cycle is basically equivalent to that presented in (Piccinelli et al. 2001). The cycle begins from the identification of some purchase needs. The buyer will conduct a survey on the e-marketplace in order to collect information about a list of suppliers that are able to fulfil this purchase need. From this list, the buyer will select a supplier and will try to achieve an agreement that satisfies both parties. If not, the buyer may choose another supplier from the list. Once an agreement has been reached, buyer and supplier sign a contract stating the precise conditions that will govern the fulfilment phase. In a final evaluation phase, the buyer will measure the performance of the supplier and this information can be used as input in future partner selections.

\subsection{The search phase}

During the search phase the challenge is how to advertise selling offers, purchase needs, and how to match these elements against each other. This is not a problem of simply defining a document format; instead, it is a problem of defining what is the information to be advertised and how it should be conveyed. For example, buyers must advertise a purchase need in such a way that it can be matched with the available selling offers. If each buyer or supplier develops its own method of advertising offers and needs, it will be virtually impossible to match and identify opportunities, and to fully use the potential of the emarketplace.

Rather than developing proprietary and ineffective approaches, one should look for existing research approaches in this field. Indeed, the European Innovation Relay Centre 
Network (CORDIS 2000), a project supported by the EU, has developed an excellent approach to tackle the challenge of expressing and identifying opportunities for small and medium-sized enterprises (SMEs). This project defines two complementary types of documents: the technology offer (TO) and the technology request (TR). The IRC Network collects TOs and TRs by means of relay centres that dispatch these documents to a central database where they are matched to each other. Whenever opportunities are identified, the relay centres will forward the corresponding TO and TR to the original enterprises (the TO being sent to the prospective buyer and the TR to the supplier).

For the purpose of P2P e-marketplaces, we are interested only in the structure and information contained in these TOs and TRs. Both of these documents are anonymous and they contain both human-readable information and data that can be processed automatically. As illustrated in figure 4, every TO or TR has a title, an abstract and a short description. It also includes a set of standard technology codes taken from a list of predefined values that range virtually every industry sector. The stage of development field is used to determine whether the product is (or should be) under development, if it is (or should be) a prototype, or if it is (or should be) readily available on the market. The intellectual property rights field, used only for TOs, is used to specify any patent rights or copyright protection that may apply. Every TO and TR also include current or desired values for the organisation type (industry, technology transfer centre, research institute, etc.), size (approximate number of employees), and the type of collaboration being sought (co-operation, joint venture, commercial agreement, license agreement, etc.). The application domains section includes standard market application codes for a wide range of sectors such as communications, electronic components, molecular biology, medicine, energy, consumer products, chemicals, transportation and finance.

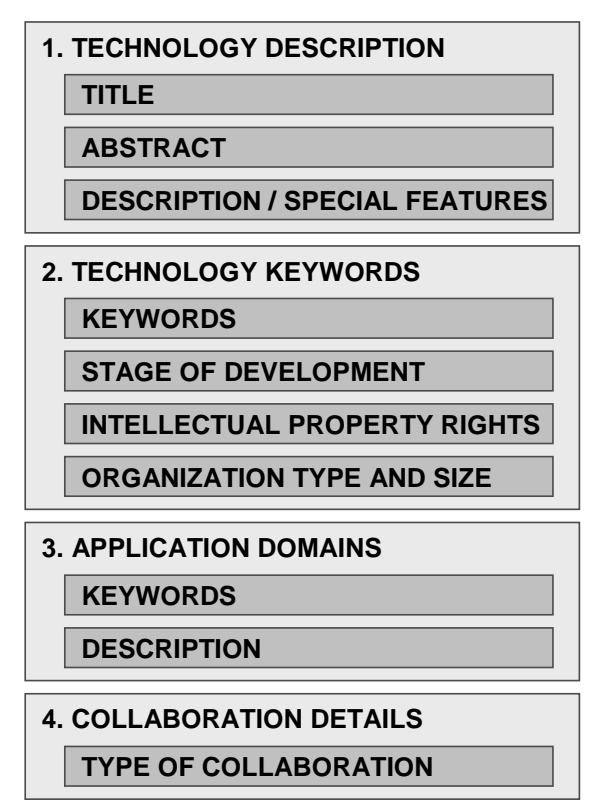

Figure 4. Structure of TOs and TRs

\subsection{The selection phase}

During the selection phase, the buyer is given a list of TOs that match the original TR according to some criteria. From the available fields, it is possible to automate the matchmaking of TOs and TRs to some degree, based on the keywords and market application codes. This automated matchmaking process will yield, for a given TR, the TOs that are positioned within the same market segment and refer to the same kind of applications. 
However, this will still produce some results that do not address the need at hand, so these entries should be pruned by means of a pre-selection of the relevant suppliers.

Once there is a set of one or more suppliers that are able to fulfil the purchase need, the buyer will engage in one-to-one conversations with those suppliers. In each conversation the identity of both buyer and supplier are disclosed. They exchange the required information - such as products, quantities, delivery method, price, and payment method - in order to ascertain the conditions for the purchase of goods, services, or technology. Once the results from these inquiries have been collected from all of the prospective suppliers, the buyer will be able to identify the supplier providing the best conditions, by considering parameters such as price, quality and service fulfilment.

\subsection{The contracting phase}

The purpose of the contracting phase is to elaborate and sign a Trading Partner Agreement (TPA) that specifies how the purchase will take place, from initial ordering to final payment. Currently, there are proposals for e-commerce-related TPA formats coming from two different backgrounds. One is the legal point of view that looks upon a TPA as a legal document that binds a buyer to a supplier according to written conditions. From this point of view, the TPA specifies several details concerning issues such as the ordering process details, the product items covered by the contract, the payment method, the delivery options and costs, performance requirements, product warranty, and the mechanisms for resolving disputes or disagreements (Taylor 2000).

The second point of view comes from a purely technological perspective. Recent approaches such as IBM's tpaML (Dan et al. 2001) and ebXML's CPA (Collaboration Protocol Agreement) (UN/CEFAT and OASIS 2001) have led to specifications with a strong technological bias. These specifications focus on defining the message exchange sequence and the particular communication protocols used for exchanging those messages, such as HTTP, SMTP or FTP, and secure transport protocols such as SSL. However, the availability of these protocols is a requirement that pertains the underlying communication infrastructure, not the purchase process that will take place between buyer and supplier. The relevant contribution of these TPA proposals lies in the ability to specify the sequence of exchanges, which is typically pre-defined in most e-business frameworks (Shim et al. 2000).

Given that all the required technology is readily available (XML, S/MIME, HTTPS, TLS, digital signatures), the challenge is to define a contractual framework that assists in developing legally supported business relationships that are accompanied by a TPA, where a TPA is the common and neutral representation for all obligations the involved parties agreed on (Merz et al. 1998). Several research projects have addressed this challenge, for example ECLIP (Cavanillas and Nadal 1999), eLEGAL (Hassan et al. 2001), Octane24 (ComNetMedia 2001), and CrossFlow (Hoffner 1999). Unfortunately, there is still little agreement concerning the contents and usage of TPAs, and there is an urgent need for standardisation in this field.

\subsection{The fulfilment phase}

During the fulfilment phase, buyer and supplier perform the exchanges they have previously contracted. For this purpose, several B2B frameworks are available nowadays, such as OBI (OBI 1999), RosettaNet (RosettaNet 1999), BizTalk (Microsoft 2000) or ebXML (UN/CEFAT and OASIS 2001), to name just a few. These frameworks try to address two main requirements: how to facilitate exchanges between buyer and supplier over the Internet, and how to integrate those exchanges with the existing information systems at each end. To meet these requirements, the frameworks rely on XML in order to define message exchange formats, though some frameworks were also inspired by EDI (e.g. OBI, ebXML). 
The large number of B2B frameworks and XML-based specifications available today means that there are a lot of B2B exchanges for which message and document formats have already been defined. These range several areas such as product information, ordering, customer service and financial exchanges. In some cases, there are even several alternative frameworks. The conclusion is that, even though there may be no B2B framework that exactly fits the requirements for a given set of buyer-supplier exchanges, it is possible to pick the appropriate exchange formats from the wide range of available B2B frameworks. In other words, there is no need to develop proprietary exchange formats. The amount of effort that was put on the development of B2B frameworks suggests that it is always possible to find a suitable document format, or one that can be easily adapted to particular exchange needs.

\subsection{The evaluation phase}

In the evaluation phase the buyer will measure key performance indicators such as purchase process duration, quality/price relation and effectiveness of customer service. Besides collecting information about the capabilities and performance of the contracted partners, the buyer will periodically probe the e-marketplace in order to determine the capabilities and performance of other suppliers for the same parts or products, and to become aware of new suppliers that have entered the market. In fact, the dynamics and competitiveness of the market - that the P2P e-marketplace gives a clearer picture of - will require suppliers to continuously improve its operational processes, and to make every effort to expand its customer base.

Gradually, as buyers and suppliers conduct exchanges through the e-marketplace, both kinds of peers will start developing trust relationships. However, due to the dynamics of the P2P e-marketplace, a new sense of trust will arise, and hence it is appropriate to distinguish between inductive trust and deductive trust (Goranson 1999). Inductive trust can be seen as the trust supported by knowledge about a given peer in continuous, stable market conditions. In dynamic markets, where peers frequently associate with different business partners in order to exploit rapidly changing opportunities, peers develop deductive trust, i.e., trust supported by knowledge about the market in dynamically changing conditions. More specifically, peers trust that they are able to find on the e-marketplace the business partners that will allow them to respond to a given market opportunity.

Enabling trust on a P2P e-marketplace is actually a complicated challenge since the decentralisation, flexibility and peer autonomy that characterise the P2P e-marketplace are prone to potential misuse. Spurious content, dubious peer intentions, verification and certification of information, quality of service, and vulnerability to security attacks (Parameswaran et al. 2001) are some of the challenges that a P2P e-marketplace must overcome by implementing mechanisms to protect its business community. To some extent, this depends on the features and capabilities of the underlying P2P infrastructure and services.

\section{Implementing P2P e-marketplace services}

Several P2P platforms have already demonstrated their potential, particularly in distributed information processing (SETI@home), file sharing (e.g. Gnutella) and instant messaging (e.g. Jabber) (Oram 2001).

For example, Gnutella is a file sharing protocol based on forwarding search requests and operating in a similar fashion to what was shown in figure 3. Freenet focuses on uncensored publishing and dissemination of documents, while protecting their contents with private keys and ensuring the anonymity of the corresponding authors. Red Rover relies on Web-based e-mail accounts to allow peers to exchange content that traverses censorship barriers, without the need to establish bi-directional connections. Jabber is a decentralised instant messaging solution that is interoperable with most existing instant messaging 
applications. In fact, several commercial products are increasingly providing P2P functionality such as instant messaging (e.g. IBM Lotus).

It becomes clear, however, that none of these platforms provides the range or depth of services required to support the B2B trading life-cycle. In addition, most of these platforms are narrowly focused on a particular purpose. Only a generic and extensible P2P platform, such as JXTA (Sun 2001), will enable he implementation of higher-level, business-oriented services.

\subsection{Project JXTA}

Project JXTA is named after "juxtaposition", the act of placing peers side by side. Project JXTA is arguably the most comprehensive and flexible P2P platform currently available. In a JXTA network, peers communicate through pipes, a protocol-independent abstraction. Pipes may use TCP/IP sockets, IP multicasting or HTTP to establish a connection between two peers on the network, no matter how far apart. A pipe is not necessarily a direct connection, but instead it is usually attained through a sequence of other peers. Special kinds of peers, such as routers and rendezvous peers, allow traffic to bypass firewalls.

Another powerful feature of JXTA is that peers may create and join peer groups where special-purpose services may be available. A JXTA service can be any functionality that implements behaviour on the P2P network. JXTA services may be used for searching, messaging, or any other purpose that peers may find useful. Peer groups may be protected by membership rules that allow only peers with certain credentials to join the group. Inside a peer group, peers implement services that may or may not be available outside that group. Therefore, a peer group is as a protected service space.

The entire JXTA infrastructure is based on XML documents called advertisements. In fact, all JXTA resources are represented by an advertisement: there are advertisements for peers, peer groups, pipes and services. JXTA already provides low-level, community services such as the DiscoveryService that enables the search for advertisements, the MembershipService that checks the credentials of a peer before allowing that peer to join the group, and the PipeService that assists in the creation of pipes between peers.

The typical peer behaviour can be described as follows. At first, the peer logs into the network, which automatically publishes its own peer advertisement. Then the peer will search for a group advertisement using the DiscoveryService. When the group advertisement is found, the peer invokes the MembershipService in order to join that group. Once inside the group, the peer will search for service advertisements, again using the DiscoveryService. Each service advertisement contains a pipe advertisement that enables peers to communicate with the peers implementing that service. After the peer extracts the pipe advertisement from the service advertisement, it uses the PipeService in order to create input and output pipes to communicate with that service.

\subsection{Building an e-marketplace on JXTA}

The B2B trading life-cycle requires two different kinds of services: multicast (one-to-many) services that allow a peer to disseminate a TR and search for matching TOs, and unicast services that allow peers to attain exchanges on a one-to-one basis. These two kinds of services correspond to the networking mechanisms shown in figures 3(a) and 3(b), respectively. For the P2P e-marketplace, we have devised one multicast service and three unicast services. These services support the first four phases of the B2B trading life-cycle.

Multicast services can be easily implemented using propagate pipes. A propagate pipe is a one-directional pipe that connects one sending peer to several receiving peers. The sender must request the PipeService to create the propagate pipe as an output pipe, while the receivers create this pipe as an input pipe. 
Search capabilities can be implemented by means of a Trading Partner Search Service (TPSS). An advertisement for TPSS is available within the peer group, and that advertisement contains an advertisement for a propagate pipe. When a buyer joins the peer group, this peer will retrieve the TPSS advertisement, and from this it will extract the pipe advertisement in order to create an output pipe. When suppliers join the peer group they perform the same actions, but they will create an input pipe rather than an output pipe. This will allow buyers to submit TRs to suppliers. If one or more suppliers find a TR that matches one of their own TOs, they will reply to the original buyer by means of a regular unicast pipe. This means that the TPSS actually comprises two pipes: a propagate pipe to submit TRs and a unicast pipe to receive matching TOs, as shown in figure 5.

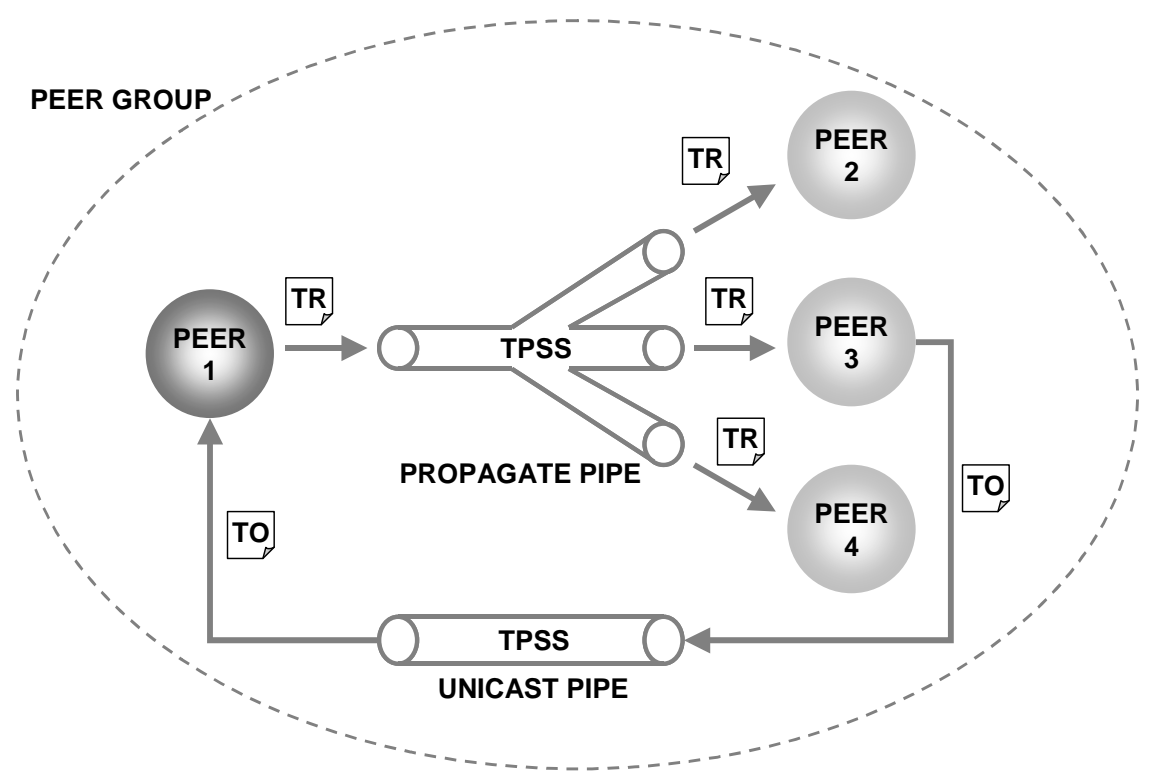

Figure 5. Multicast e-marketplace service (search phase)

Once a buyer has retrieved a list of proposed TOs from suppliers, unicast services come into play. Should one or more TOs be of interest to the buyer, this peer will initiate a new set of exchanges with the corresponding suppliers. At first, the buyer will want to know who the potential suppliers are (since TOs are anonymous), so the Trading Partner Information Service (TPIS) will allow the buyer to exchange company profile information with the potential supplier. Because the TPIS is a generic information exchange service, there is no mandatory format for the information exchanged. Its aim is to provide human-readable information about the each party, and to serve as a decision-support service during the selection phase.

The TPIS is available as a service advertisement that contains a unicast pipe advertisement. When retrieving the service advertisement, both parties extract the pipe advertisement and both create an input and output pipe that will allow them to exchange information bi-directionally, on a request-reply basis. Unicast pipes are secure communication channels since they make use of TLS (Transport Layer Security) functionality provided by JXTA. 


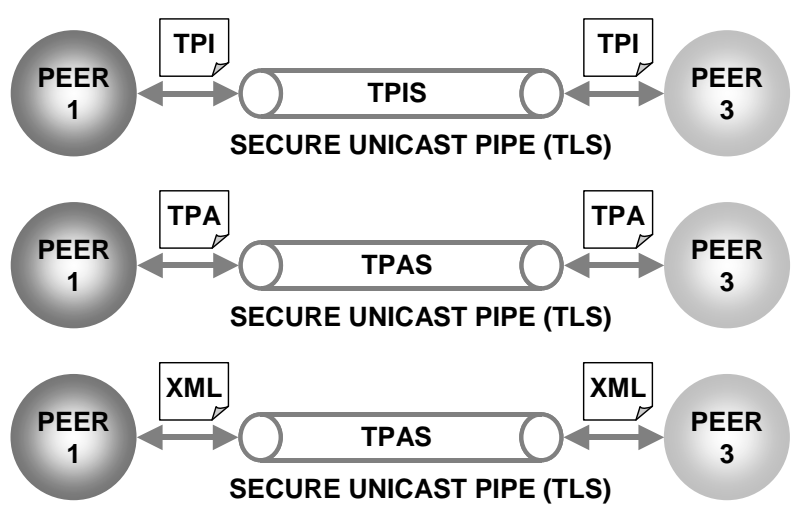

Figure 6. Unicast e-marketplace services (selection, contracting, and fulfilment phases)

The exchange of profile information is the first step that will eventually lead to the establishment of a contract between both parties. This means that, should the buyer be interested in the supplier's offer, a series of negotiation exchanges will take place between those peers. Hence, every TPIS message should include a new pipe advertisement, this time for interacting with a contract-supporting service, the Trading Partner Agreement Service (TPAS). This way, and after the selection phase is concluded, the chosen supplier can be contacted in order to develop a TPA. The TPAS uses a secure unicast pipe just like the TPIS but, in contrast with the request-reply behaviour of TPIS, the TPAS allows iterated exchanges until a (digitally signed) TPA has been achieved.

According to the same logic, a TPA includes the pipe advertisements needed for the fulfilment phase. Through these pipes, local applications at each peer can communicate directly, sending from or bringing messages to the local information system. This is achieved through the Trading Partner Exchange Service (TPES) that supports exchanges of arbitrary documents, possibly according to one or more B2B frameworks.

\section{A P2P e-marketplace for industrial equipment}

In order to assess the acceptance and practical use of a P2P e-marketplace, we have prepared a laboratory scenario for a vendor of industrial equipment. This vendor is a Portuguese company involved in the design, development, manufacture, installation and maintenance of machinery and spare parts for the shoe industry. The company also provides industrial equipment for sofa and settee production lines, and for several metal mechanics production units. Its business goal is to provide a wide range of solutions that will enable installation, maintenance and upgrade of any type of high quality automated production system. The company's main revenues come from selling and installing equipment that it designs and produces. Additional income comes from maintenance, upgrade, and training personnel at customers' sites.

In recent years, the characteristics of its market and the constant competitive pressure have forced the company to adapt to market conditions. Today, the company's strategy focuses on new industrial sectors, on profitable customer services, on facilitating relationships with suppliers and resellers, on enhancing maintenance processes, and on the increase of its international presence and expansion to new markets. The expansion into new markets requires the company to find resellers that are able to deal with local customers. This is particularly imperative regarding maintenance, where there is a need to guarantee QoS parameters such as timely intervention.

The challenges the company faces, together with a resolute determination towards improving its market position in an increasing global market, have led the company to invest considerably on research projects and co-operation with research centres. The following 
paragraphs briefly summarise a hypothetical scenario in which the company and its customers, suppliers and resellers become members of a P2P e-marketplace. The purpose of this study was to show multicast and unicast services in action, and to illustrate the possibilities of the P2P approach. The implementation of this scenario, however, will only be possible when the e-marketplace reaches a certain critical mass, which requires other members, beyond the company and its business partners, to participate.

\subsection{Supporting customer relationships}

The company produces, installs and provides maintenance for industrial equipment that is highly tailored or customised according to customer needs. However, the company only commits itself to producing equipment that has potential for being sold to other customers. When it gets into contact with a new potential customer, the company shows a catalogue of previously developed equipment. The purpose of this catalogue is not necessarily to identify a product that fits the customer's needs, but to exhibit a set of products that represent the kind of solutions the company provides.

The TOs to be developed for this company must include, therefore, a reference to these solutions in section 1 of figure 4. Other elements in section 2 will refer to the development stage of those solutions, to any patent rights that apply, to the company type (industry, in this case) and its size (11 to 50 employees). The technology keywords correspond to the technology the company provides and must be taken from the industrial manufacturing section of standard technology keywords. The application domain keywords refer to the keywords that are most akin to the target industry sectors (shoe manufacturing, sofa and settee production lines, metal mechanics). Finally, the type of collaboration could be defined as a "commercial agreement with technical assistance".

When developing the TOs it is possible that there are not sufficiently detailed technology keywords to describe this vendor's solutions. One should note, however, that TOs are to be matched against TRs from potential customers searching for industrial equipment. Thus, TOs should not be too specific or too detailed, or so narrowly focused that they would possibly fail to match certain customer needs that this vendor can indeed address. Instead, because the company is able to produce highly customized equipment, the TOs should be as generic as possible, though mentioning all the technology this vendor is able to provide.

Whenever a TR is submitted through the TPSS service, regardless of whether it matches this vendor's TOs or not, the company can keep that TR in a cache in order to evaluate market needs and opportunities. When a TR does match a local TO, the TPSS service running on the company will send the TO back to the potential customer. If the customer is interested in the TO, unicast services take over from this point. TPIS allows the company to get to know the customer as well as its needs and expectations. It allows the company also to determine if the customer needs can be fulfilled by one of the catalogue solutions, or if a new product must be developed. In this case, the company evaluates the potential for this new product to be sold in the market. For this purpose, the company may refer to the TRs in its cache, to help in estimating market acceptance for this new product.

If the product is both feasible and likely to be profitable, the company will accept the order and establish a contract with the customer, using the TPAS service. It will then initiate a set of parts purchasing and internal production processes. During this phase, exchanges between the company and the customer, such as inquires and plant specifications, are attained through TPES. The TPAS will be eventually used once again with the same customer in order to establish a maintenance contract after installation. 


\subsection{Supporting parts purchasing}

When the company accepts the customer order and commits itself to the production of the industrial equipment, the first step is to generate the complete material and parts list. Some of these parts and materials are available from internal stock, others are purchased from an already contracted supplier, and yet for others a supplier must be found. In this last case, the company writes a TR for each kind of material or part and submits the TRs to the e-marketplace using the TPSS service. Matching TOs will then be considered, and a preselection of potential suppliers takes place. The company then sends each of the potential suppliers a request for quotation (RFQ) using the TPIS service.

After the suppliers are selected, a price list for materials and parts is compiled and the company has to go through an internal step of requisition approval. Once approval is granted, the company sets the conditions with the suppliers by means of contracts, using TPAS, and submits the corresponding orders using TPES. These orders include both purchase needs for production, and spare parts quantities to be kept in stock and to be used for maintenance purposes. Contracts with suppliers may include subsequent purchase of further quantities. Delivery notes and invoices are exchanged using TPES.

\subsection{Supporting company expansion: the inverse search problem}

Installation and maintenance are resource-intensive and time-consuming tasks that can become even more demanding when the customer is in another country. In this case, and when additional opportunities have been identified in a certain geographical location, it is more appropriate and effective for the company to be represented by a local reseller. This reseller provides customer service (installation, maintenance and training) to the customer base on that location. In order to search for resellers, the company develops and disseminates a TO specifying the same technology as the TO for customers, but using different collaboration requirements. This time the TPSS performs a search based on a TO, matching the offer against the available TRs at each peer. This is called inverse search, since it is equivalent to a supplier searching the e-marketplace for buyers.

Table 2 summarises the use of multicast and unicast services in this industrial equipment e-marketplace.

\begin{tabular}{|c|c|c|}
\hline Trading phase & $\begin{array}{c}\text { E-marketplace } \\
\text { service }\end{array}$ & Relevant data \\
\hline Customer performs search & TPSS & TO \\
\hline $\begin{array}{l}\text { Customer information and } \\
\text { order information }\end{array}$ & TPIS & $\begin{array}{l}\text { Customer TR, product catalogue, } \\
\text { and product description }\end{array}$ \\
\hline Product feasibility analysis & (internal services) & $\begin{array}{l}\text { Cached TRs, internal cost/profit } \\
\text { analysis }\end{array}$ \\
\hline $\begin{array}{l}\text { Contract establishment with } \\
\text { customer }\end{array}$ & TPAS & Selling contract \\
\hline Search for supplier & TPSS & TR \\
\hline Supplier selection & TPIS & Supplier TOs, RFQ \\
\hline $\begin{array}{l}\text { Contract establishment with } \\
\text { supplier }\end{array}$ & TPAS & Buying contract \\
\hline Parts purchasing & TPES & $\begin{array}{l}\text { Parts orders, delivery notes, } \\
\text { invoices }\end{array}$ \\
\hline Internal production & (internal services) & $\begin{array}{l}\text { CAD design, implementation } \\
\text { specifications }\end{array}$ \\
\hline $\begin{array}{l}\text { Installation at customer's } \\
\text { site }\end{array}$ & TPES & $\begin{array}{l}\text { Installation requirements and } \\
\text { guidelines }\end{array}$ \\
\hline $\begin{array}{l}\text { Establish contract for } \\
\text { maintenance }\end{array}$ & TPAS & Maintenance contract \\
\hline Maintenance intervention & TPES & Failure assessment, upgrade \\
\hline
\end{tabular}




\begin{tabular}{lll}
\hline & & requirements \\
\hline Search for reseller & TPSS & TO (inverse search) \\
\hline Reseller selection & TPIS & Reseller TRs, reseller location \\
\hline $\begin{array}{l}\text { Contract establishment with } \\
\text { reseller }\end{array}$ & TPAS & Reseller contract \\
$\begin{array}{l}\text { Outsourced installation and } \\
\text { maintenance }\end{array}$ & TPES & $\begin{array}{l}\text { Product data sheets, maintenance } \\
\text { data, spare parts needs }\end{array}$ \\
\hline
\end{tabular}

Table 2. Service usage in the industrial equipment e-marketplace

\section{Conclusion}

The traditional e-marketplace, built according to Web-based client-server architectures exhibits several limitations mainly because it is a centralised solution for a problem which requires a distributed infrastructure. On the other hand, the impact and success of completely decentralised P2P solutions has placed this paradigm as a meaningful alternative to support B2B trading. Recently, several P2P infrastructures have been proposed (Gnutella, FreeNet, JXTA, Jabber, etc.). Still, these infrastructures lack essential services allowing companies to use them as a business platform. These services are of two kinds:

- Multicast services support one-to-many interaction and are suitable for partner search, either buyers searching for suppliers, or suppliers searching for buyers (inverse search). These services are implemented inside membership-protected peer groups.

- Unicast services support one-to-one interaction and are suitable for inquiries, negotiation, contract establishment and implementation of general B2B exchanges. These services require security features such as encrypted communication channels and digital signatures.

Despite the broad scope and inter-disciplinary nature of these $\mathrm{P} 2 \mathrm{P}$ services, the conclusion is that all of the required technology is presently available. However, advances in e-contracting and trading partner agreements are necessary in order to establish some standardised approaches in this area.

Whereas the traditional e-marketplace is appropriate for trading commodities, the P2P e-marketplace supports one-of-a-kind products as well, as the application scenario has shown. As a consequence, the P2P e-marketplace can be used in virtually any industry or commercial sector where TOs and TRs, in the present form or another, can be used to express selling offers and purchase needs, respectively.

The P2P e-marketplace allows peers to implement business strategies such as customer relationship management (CRM) by enabling one-to-one exchanges of run-time information between buyers and suppliers. The P2P e-marketplace is also an enabler for higher-level architectures that are strongly based on trust such as, for example, automatic negotiation and multi-agent systems. Thus, the P2P e-marketplace fosters the outgrowth of both current and new, forthcoming business integration approaches.

Acknowledgment. The first author highly appreciates the support of the Fundação para a Ciência e a Tecnologia (http://www.fct.mct.pt), which is funding his research.

\section{References}

Cavanillas, S., Nadal, A., 1999, Deliverable 2.1.7: Contract Law, http:/www.eclip.org/ documents/deliverable_2_1_7_contract_law.pdf

ComNetMedia AG, 2001, OCTANE e-contracting service concept, http://www.octane24.com/ 
CORDIS, 2000, IRC Public Website, http://irc.cordis.lu/

Dan, A., Dias, D., Kearney, R., Lau, T., Nguyen, T., Parr, F., Sachs, M., Shaikh, H., 2001, Business-to-business integration with tpaML and a business-to-business protocol framework. IBM Systems Journal, 40(1), pp. 68-90

Goranson, H. T., 1999, The Agile Virtual Enterprise: Cases, Metrics, Tools (Quorum Books)

Hassan, T., Carter, C., Hannus, M., Hyvärinen, J., 2001, eLEGAL: Defining a Framework for Legally Admissible Use of ICT in Virtual Enterprises. Proceedings of the 7th International Conference on Concurrent Enterprising (ICE2001), Bremen, Germany, 27-29 July, pp. 347355

Hoffner, Y., 1999, Supporting Contract Match-Making. Proceedings of the 9th International Workshop on Research Issues on Data Engineering (RIDE-VE'99), Sydney, Australia, 23-24 March

Merz, M., Griffel, F., Tu, T., Müller-Wilken, S., Weinreich, H., Boger, M., Lamersdorf, W., 1998, Supporting Electronic Commerce Transactions with Contracting Services. International Journal of Cooperative Information Systems, 7(4), pp.249-274

Microsoft, 2000, The BizTalk ${ }^{\mathrm{TM}}$ Framework, http://www.biztalk.org/

Norris, M., West, S., 2001, eBusiness Essentials: Technology and Network Requirements for Mobile and Online Markets (John Wiley \& Sons)

OBI Consortium, 1999, Open Buying on the Internet: Technical Specifications, version 2.0, http://www.openbuy.org/obi/specs/

Oram, A. (Ed.), 2001, Peer-to-Peer: Harnessing the Power of Disruptive Technologies (O’Reilly \& Associates)

Parameswaran, M., Susarla, A., Whinston, A., 2001, P2P Networking: An InformationSharing Alternative. IEEE Computer, 34(7), pp.31-38

Piccinelli, G., Stefanelli, C., Morciniec, M., Casassa-Mont, M., 2001, Policy-based Management for E-Service Delivery. HP OpenView University Association (HP-OVUA) $8^{\text {th }}$ Annual Workshop, Berlin, June 24-27

RosettaNet Inc., 1999, Guide to RosettaNet Specifications, http://www.rosettanet.org/

Shim, S., Pendyala, V., Sundaram, M., Gao, J., 2000, Business-to-Business E-Commerce Frameworks. IEEE Computer, 33(10), pp. 40-47

Sun Microsystems, 2001, Project JXTA, http://www.jxta.org/

Taylor, M. L., 2000, TPA Sample, http://www.mltweb.com/ec/tpa.htm

UN/CEFACT and OASIS, 2001, ebXML Technical Architecture Specification, http://www.ebxml.org/specs/ebTA.pdf 